\title{
PAPER \\ Time-Reversal MUSIC Imaging with Time-Domain Gating Technique
}

\author{
Heedong CHOI ${ }^{\dagger a)}$, Nonmember, Yasutaka OGAWA ${ }^{\dagger}$, Fellow, Toshihiko NISHIMURA ${ }^{\dagger}$, \\ and Takeo OHGANE ${ }^{\dagger}$, Members
}

\begin{abstract}
SUMMARY A time-reversal (TR) approach with multiple signal classification (MUSIC) provides super-resolution for detection and localization using multistatic data collected from an array antenna system. The theory of TR-MUSIC assumes that the number of antenna elements is greater than that of scatterers (targets). Furthermore, it requires many sets of frequencydomain data (snapshots) in seriously noisy environments. Unfortunately, these conditions are not practical for real environments due to the restriction of a reasonable antenna structure as well as limited measurement time. We propose an approach that treats both noise reduction and relaxation of the transceiver restriction by using a time-domain gating technique accompanied with the Fourier transform before applying the TR-MUSIC imaging algorithm. Instead of utilizing the conventional multistatic data matrix (MDM), we employ a modified MDM obtained from the gating technique. The resulting imaging functions yield more reliable images with only a few snapshots regardless of the limitation of the antenna arrays.

key words: time-reversal (TR) technique, TR-MUSIC imaging, ultra wideband $(U W B)$, gating technique
\end{abstract}

\section{Introduction}

One promising candidate for locating and detecting obscured scatterers (targets) is time-reversal (TR) imaging [1]. TR techniques exploiting the invariance of the wave equation for remote sensing were initially developed for use in acoustics [1], [2]. Later, they were applied to electromagnetic waves using time-harmonic waves and a time reversal array (TRA) [3]-[13]. Due to the classical diffraction limit, the focusing spot size (focusing region) in conventional imaging techniques is severely restricted by the antenna aperture length. TR-based techniques, on the other hand, provide better focusing resolution by effectively increasing the antenna aperture length [13]. The developed TR imaging methodology conducts eigenvalue decomposition of the time reversal operator (TRO) obtained from a multi-static data matrix (MDM) by a limited-aspect antenna array. For well-resolved (orthogonal) point-like scatterers, the conventional decomposition of the TRO works well because each eigenvalue corresponds to each scatterer. Unfortunately, the performance of TR-based techniques greatly deteriorates if this resolution condition is not met and/or the antenna array is sparse [5].

A time-reversal multiple signal classification (TR-

Manuscript received September 5, 2011.

Manuscript revised March 9, 2012.

†The authors are with the Graduate School of Information Science and Technology, Hokkaido University, Sapporo-shi, 0600814 Japan.

a) E-mail: hdchoi@m-icl.ist.hokudai.ac.jp

DOI: 10.1587/transcom.E95.B.2377
MUSIC) technique has been proposed to overcome the problem of inadequate resolution. TR-MUSIC imaging provides super-resolution for detection and localization by using the noise subspace of the TRO, which is orthogonal to the signal subspace [5]. Studies on TR imaging have been conducted, such as general configuration [3] and extension to multiple (non-point-like) targets [9], multiple scattering scenarios [4], [7], measurements and experiments in various background media [6], [8], single-frequency [5] and ultrawideband (UWB) signals [10], a variety of matrices of the space-space [5] and the space-frequency [11], and imaging with a single antenna [12].

Theoretically, any TR-MUSIC technique must satisfy the prerequisite of providing more transceivers $N$ than targets $M(N>M)$. Regretfully, it may be difficult to satisfy this condition because we cannot have a large number of antenna elements. In addition, the TR-MUSIC technique necessitates many sets of frequency-domain data (snapshots) in heavy noise environments since the received signals are largely corrupted by noise. However, it is not preferable to require many snapshots because a long measurement time is required.

To overcome the above obstacles, we have proposed the basic concept of a TR-MUSIC imaging approach using a time-domain gating technique, and conducted preliminary performance evaluations [15], [16]. In this paper, we address the formulation of our approach, and discuss its performance in more detail. The concept of time-domain gating has been examined for radio frequency measurement fields such as antenna measurement [14]. Measured frequencydomain data are transformed into time-domain ones with the inverse Fourier transform. Time-domain gating can mathematically eliminate unwanted signal responses using a gating filter (window) in the time domain. This technique can also reduce random noise components outside the gate. By applying the Fourier transform to the gated time-domain data, we obtain frequency-domain data that have no contribution of signals and noise components outside the timedomain gate. When the time-domain gating technique is used in TR-MUSIC, the response resolution of the inverse Fourier transform depends on the frequency bandwidth of the measured data. Thus, it is difficult to resolve received signals in traditional narrowband cases due to the limited range resolution. Recent developments in UWB provide the potential to surpass the conventional range limitation by means of unprecedented resolution capability. If we extract 
time-domain response(s) with wideband data around a certain time without damaging the target signal(s) by using gating, we can not only suppress the noise components but also eliminate target response(s) outside gating filter (window) in the time domain. This can increase the signal to noise ratio (SNR). In addition, we can obtain a new MDM that has contributions from fewer targets. If we choose an adequate gate width, we have fewer targets than the number of antennas. In other words, if we use the modified MDM obtained from the time-domain gating technique instead of using the conventional MDM, we can simultaneously improve the performance of TR-MUSIC imaging and overcome the restricted condition. We repeat this TR imaging process by changing the center time of the gate. We can then obtain the TR imaging of the entire region. The process combining timedomain gating and TR-MUSIC is relatively simple, but it is considerably effective in performance enhancement by eliminating undesirable effects such as noise and response(s).

The remainder of this paper is organized as follows. First, we briefly introduce the TR process and the formulation of the TR-MUSIC imaging technique in Sect. 2. In Sect. 3, we formulate the problem of TR-MUSIC and propose a super-resolution technique developed with the gating technique. In Sect. 4, we discuss the performances of the proposed TR-MUSIC approach using computer simulation results. Finally, we give conclusions in Sect. 5.

\section{Overview of TR-MUSIC Imaging}

In this section, we review the TR-MUSIC imaging technique for multiple scatterers. The developed TR-MUSIC uses the eigenvalue decomposition of the TRO obtained from the MDM. Due to utilization of its null steering, the TR-MUSIC imaging technique provides better detection and localization properties in non-well-resolved cases as well as well-resolved ones.

An $N$ element TRA produces an $N \times N$ symmetric (due to the reciprocity principle) MDM denoted as $\mathbf{K}(\omega)=$ $\left\{k_{i, j}(\omega)\right.$ for $\left.i, j=1,2, \ldots, N\right\}$, where $\omega$ is an angular frequency of the wave. Bold letters denote vectors or matrices. The MDM is obtained as follows: we send a signal from the first element in the TRA and record the reflected waveform in each TRA element to obtain the first column of the MDM. We repeat this process to the end of the TRA to obtain all the columns. Using the obtained MDM, the TRO is defined as a self-adjoint matrix: $\mathbf{T}(\omega)=\mathbf{K}^{\dagger}(\omega) \mathbf{K}(\omega)$, where the superscript $\dagger$ denotes the Hermitian conjugate. We then apply singular value decomposition (SVD) to the MDM to obtain: $\mathbf{K}(\omega)=\mathbf{U}(\omega) \boldsymbol{\Lambda}(\omega) \mathbf{V}^{\dagger}(\omega)$, where $\mathbf{U}(\omega)$ and $\mathbf{V}(\omega)$ are unitary matrices, $\boldsymbol{\Lambda}(\omega)$ is a diagonal and real-valued matrix with singular values $\lambda_{1}(\omega), \lambda_{2}(\omega), \ldots, \lambda_{N}(\omega)$. Similarly, we can rewrite the TRO using SVD of the MDM: $\mathbf{T}(\omega)=\mathbf{V}(\omega) \mathbf{S}(\omega) \mathbf{V}^{\dagger}(\omega)$, where $\mathbf{S}(\omega)=\boldsymbol{\Lambda}^{\dagger}(\omega) \boldsymbol{\Lambda}(\omega)$ is the realvalued diagonal matrix with eigenvalues $\lambda_{1}^{2}(\omega), \lambda_{2}^{2}(\omega), \ldots$, $\lambda_{N}^{2}(\omega)$. The eigenvectors of the TRO coincide with the columns of the unitary matrix $\mathbf{V}(\omega)$, which are composed of $\mathbf{v}_{i}(\omega)$ for $i=1,2, \ldots, N$. Therefore, the signal subspace
$S S$ of the TRO is spanned by eigenvectors $\mathbf{v}_{1}(\omega), \mathbf{v}_{2}(\omega)$, $\ldots, \mathbf{v}_{M_{S}}(\omega)$ with significant eigenvalues $\lambda_{1}^{2}(\omega), \lambda_{2}^{2}(\omega), \ldots$, $\lambda_{M_{S}}^{2}(\omega)$, and the noise subspace $N S$ is spanned by $\mathbf{v}_{M_{S}+1}(\omega)$, $\mathbf{v}_{M_{S}+2}(\omega), \ldots, \mathbf{v}_{N}(\omega)$, where $M_{S}$ is the number of significant eigenvalues. For point-like scatterers in homogeneous media, the number of targets $M$ is identical to that of significant eigenvalues $M_{S}\left(M=M_{S}\right)$ [1]. In the case where there is almost no noise or SNR is very high, the signal subspace $S S$ is formed by the eigenvectors having non-zero eigenvalues whereas the noise subspace $N S$ is formed by the eigenvectors having almost zero eigenvalues, i.e.,

$$
\begin{aligned}
& S S=\left\{\mathbf{v}_{1}(\omega), \mathbf{v}_{2}(\omega), \ldots, \mathbf{v}_{M}(\omega)\right\} \\
& \text { with } \lambda_{1}(\omega)>\lambda_{2}(\omega)>\ldots>\lambda_{M}(\omega)>0 \\
& N S=\left\{\mathbf{v}_{M+1}(\omega), \mathbf{v}_{M+2}(\omega), \ldots, \mathbf{v}_{N}(\omega)\right\} \\
& \text { with } \lambda_{M+1}(\omega) \approx \lambda_{M+2}(\omega) \approx \ldots \approx \lambda_{N}(\omega) \approx 0 .
\end{aligned}
$$

Since the noise subspace NS is always orthogonal to the signal subspace $S S$, the Green's function vectors corresponding to the scatterers must be orthogonal to the noise subspace NS. This is the basis of the TR-MUSIC algorithm and provides the following imaging pseudo-spectra (imaging function) expressed in the form [5], [10]

$$
D\left(\mathbf{x}_{p}, \omega\right)=\left(\sum_{i=M+1}^{N} \mathrm{~K} \mathbf{g}\left(\mathbf{x}_{p}, \omega\right), \mathbf{v}_{i}^{*}(\omega)>\mid\right)^{-1},
$$

where $\mathbf{x}_{p}$ represents the search point vector, the angular brackets $<\cdot>$ represent the inner product, the superscript asterisk * denotes the complex conjugate, and

$$
\begin{array}{r}
\mathbf{g}\left(\mathbf{x}_{p}, \omega\right)=\left[G\left(\mathbf{x}_{p}, \boldsymbol{\alpha}_{1}, \omega\right), G\left(\mathbf{x}_{p}, \boldsymbol{\alpha}_{2}, \omega\right),\right. \\
\left.\ldots, G\left(\mathbf{x}_{p}, \boldsymbol{\alpha}_{N}, \omega\right)\right]^{T}
\end{array}
$$

is the background steering vector, which is also called the background Green's function vector since $G\left(\mathbf{x}_{p}, \boldsymbol{\alpha}_{i}, \omega\right)$ is the Green's function, where $\boldsymbol{\alpha}_{i}(i=1,2, \ldots, N)$ denotes the position of the $i$ th antenna element, and the superscript ${ }^{T}$ denotes the transpose. The Green's function is expressed as

$$
G\left(\mathbf{r}, \mathbf{r}^{\prime}, \omega\right)=\frac{1}{4 \pi\left\|\mathbf{r}-\mathbf{r}^{\prime}\right\|} \cdot \exp \left(\frac{-j \omega}{c}\left\|\mathbf{r}-\mathbf{r}^{\prime}\right\|\right),
$$

where $\mathbf{r}$ and $\mathbf{r}^{\prime}$ are the positions of the ends of a propagation path, $\|\cdot\|$ denotes the Euclidean norm, and $c$ denotes the propagation speed. The above equation holds when all antenna elements are isotropic, and also have the same and constant frequency characteristics of gain (or pattern) over the considered frequency band. In this paper, we assume that these conditions are met.

\section{Formulation of Proposed TR-MUSIC Imaging Ap- proach}

In this section, we elaborate on our super-resolution approach that not only reduces the noise component but also enables TR-MUSIC imaging to detect more scatterers than the number of the antenna elements. It is well known that 

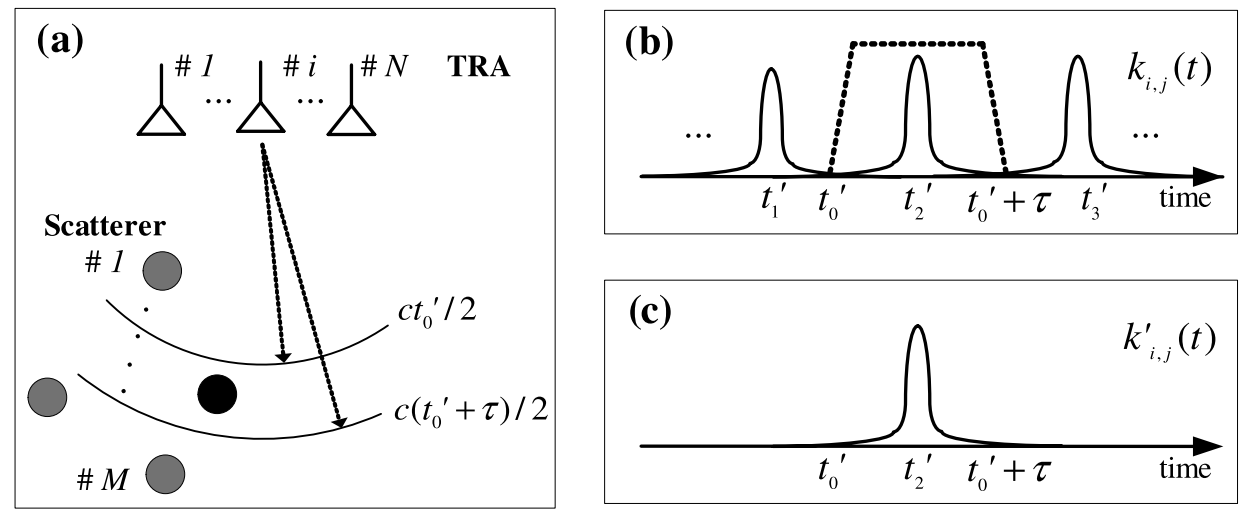

Fig. 1 Concept of the time-domain gating technique. (a) schematic illustration of a remote sensing system (b) raw time-domain responses (c) gated time-domain response.

the time-domain gating technique selects a region of interest in the time domain, eliminating unwanted responses by applying a window function. The gated response in the time domain can be transformed to the frequency domain by using the FFT without affecting the other responses in the time domain [14]-[16].

The concept of time-domain gating is demonstrated in Fig. 1. Figure 1(a) illustrates an example of a remote sensing system. We assume that there are $M$ point-like scatterers. We also assume that multiple reflections are negligibly small; therefore, each TRA element receives $M$ signal responses. In this case, each time-domain element of the MDM includes a combination of the reflected signals from all the targets. The signals concentrate around their time delay. For example, if the propagation delays of three signals are $t_{1}^{\prime}, t_{2}^{\prime}$, and $t_{3}^{\prime}$, the inverse Fourier transformed responses converge around $t=t_{1}^{\prime}, t=t_{2}^{\prime}$, and $t=t_{3}^{\prime}$, as shown in Fig. 1(b). We can extract the time-domain response at $t_{2}^{\prime}$ by using the window function from $t_{0}^{\prime}$ to $t_{0}^{\prime}+\tau$, as depicted in Fig. 1(c). This corresponds to the extraction of the target located at the position between $c t_{0}^{\prime} / 2$ and $c\left(t_{0}^{\prime}+\tau\right) / 2$, as shown in Fig. 1(a).

Next, we discuss the procedures for the proposed approach and the effect. We first obtain the frequency-domain data with $L$ equally-spaced frequencies using the TRA. The signal responses $k_{i, j}\left(\omega_{l}\right)$ in the $\operatorname{MDM} \mathbf{K}\left(\omega_{l}\right)$ for the $l$ th angular frequency can be represented as

$$
\begin{array}{r}
k_{i, j}\left(\omega_{l}\right)=\sum_{m=1}^{M} \rho_{m}\left(\omega_{l}\right) G^{*}\left(\boldsymbol{\alpha}_{i}, \mathbf{x}_{m}, \omega_{l}\right) G\left(\mathbf{x}_{m}, \boldsymbol{\alpha}_{j}, \omega_{l}\right), \\
(l=1,2, \ldots, L)
\end{array}
$$

where $\rho_{m}\left(\omega_{l}\right)$ is the scattering coefficient of the $m$ th target for $m=1,2, \ldots, M$. By means of the IFFT, we transform the frequency-domain response $k_{i, j}\left(\omega_{l}\right)$ into the time-domain response $k_{i, j}\left(t_{p}\right)$. We assume that the received signals have different time delays. They then concentrate around the time of the corresponding delay. When the sampling frequency separation is $\delta f$, the time-domain unambiguous region is given by $1 / \delta f$. Then, the noise component spreads over the entire region $1 / \delta f$.
After the transformation, we extract response(s) in a certain time region by applying the gating technique to the time-domain response $k_{i, j}\left(t_{p}\right)$. The gate width (gate span) is determined in such a way that the number of target responses in the region is less than that of antenna elements $N$. A too narrow gate span damages the signal components, whereas a too wide gate span is less effective for the noise reduction described below. Moreover, the gate shape needs to include desired target response(s) without damaging the mainlobe(s). Therefore, the window function should differ depending on the scattering scenario. By using the gating technique, the noise component outside the window is suppressed. If we use a rectangular window function with width $T_{g}$, the noise outside the gate is completely suppressed. Then, the noise power is reduced by $T_{g} /(1 / \delta f)=T_{g} \cdot \delta f$. We represent the gated response as $k_{i, j}^{\prime}\left(t_{p}\right)$ for $i, j=1,2, \ldots, N$, as shown in Fig. 1(c). However, if the gating function removes a certain amount of the response(s) of interest or it cannot suppress the unwanted response(s) almost perfectly, the gating technique is not effective and the performance of TR-MUSIC imaging deteriorates. This is the reason we need wideband data.

Next, we transform the gated response $k_{i, j}^{\prime}\left(t_{p}\right)$ into the frequency-domain response $k_{i, j}^{\prime}\left(\omega_{l}\right)$ using the FFT, and create the gated $\operatorname{MDM} \mathbf{K}^{\prime}\left(\omega_{l}\right)$. Compared to the original frequency-domain data $\mathbf{K}\left(\omega_{l}\right)$, the new frequency-domain response $\mathbf{K}^{\prime}\left(\omega_{l}\right)$ not only has much less noise components but also consists of fewer target responses than the antenna elements. Finally, we apply the TR-MUSIC imaging technique to the modified MDM $\mathbf{K}^{\prime}\left(\omega_{l}\right)$. We repeat this process for the entire area by changing the gating region. We can expect that improvement in the SNR generates more reliable images. The block diagram of the proposed approach is shown in Fig. 2.

\section{Performance Evaluations}

In this section, we show results of computer simulations of our TR-MUSIC imaging approach described in the previous section. For the performance evaluation, we considered 


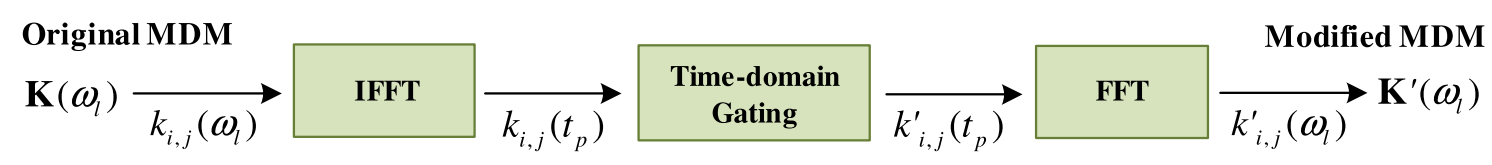

Fig. 2 The block diagram generating the modified MDM using the time-domain gating technique.
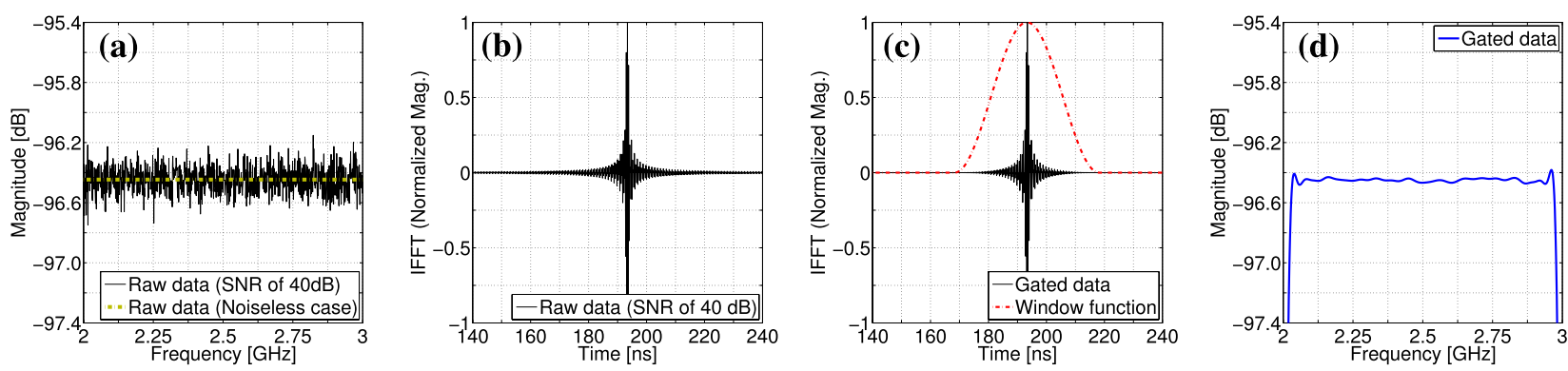

Fig. 3 Noise reduction by the gating technique. (a) raw data in the frequency domain (b) raw data in the time domain (c) gated data in the time domain (d) gated data in the frequency domain.

the following two scattering scenarios: (a) two scatterers are closely located in the time domain; thus, the gating technique cannot resolve the responses, and (b) the number of antenna elements $N$ is less than that of scatterers $M(N<M)$; therefore, the conventional TR-MUSIC imaging approach cannot work.

\subsection{Effect of Noise Reduction on TR-MUSIC Imaging}

We first discuss in detail the noise reduction effect of the gating technique. For verifying the effectiveness of our approach, 2-D computer simulations were conducted under the following conditions. The frequency band was from 2 to $3 \mathrm{GHz}$ (center frequency $f_{c}=2.5 \mathrm{GHz}$ ), and the sampling frequency separation was $\delta f=1 \mathrm{MHz}$. The linear TRA of $N=11$ was laid parallel to the $\mathrm{x}$-axis and the antenna elements were separated by $\lambda_{c} / 2$, where $\lambda_{c}$ corresponds to the wavelength at the center frequency $f_{c}$. The location of the central antenna was assumed to be $(15 \mathrm{~m}, 1 \mathrm{~m})$. Two scatterers located at $(14.5 \mathrm{~m}, 30 \mathrm{~m})$ and $(15.5 \mathrm{~m}, 30 \mathrm{~m})$ had the same scattering coefficients $\rho_{m}=1(m=1,2)$. We considered cases of SNR from 30 to $60 \mathrm{~dB}$. The signal power is defined as that of the reflection from the target at $(14.5 \mathrm{~m}$, $30 \mathrm{~m}$ ). Also, we assumed that noise is white Gaussian and has the same power over all the antenna outputs in this paper. SNR was defined as the signal-to-noise power ratio at each antenna output. The imaging function for TR-MUSIC was obtained for the center frequency $f_{c}$. Figure 3 shows the frequency and time-domain responses of $k_{1,1}$ with a single snapshot for an SNR of $40 \mathrm{~dB}$. As can be observed in Fig. 3(a), the signal level in the frequency domain ranges from around -96.8 to around $-96.1 \mathrm{~dB}$ because of the noise component. It should be noted that the signal level is given by $20 \log \left|k_{1,1}\left(\omega_{l}\right)\right| \mathrm{dB}$. Thus, if $\left|k_{1,1}\left(\omega_{l}\right)\right|=1$, the signal level is $0 \mathrm{~dB}$. Since the scatterers are located close together, the two time-domain responses are not resolved, as shown in Fig. 3(b). From the time-domain response, the gate parameters were determined as the gate center $T_{0}=193.3 \mathrm{~ns}$ and the gate span $T_{g}=50 \mathrm{~ns}$. We also used the window function type of the Raised Cosine with the roll-off $=1$, as shown in Fig. 3(c). Due to the sampling frequency separation $\delta f=$ $1 \mathrm{MHz}$, the noise component distributes over $1 \mu \mathrm{s}$. We then can suppress the noise outside the gate by using the timedomain gating technique. As can be seen in Fig. 3(d), the noise decreased due to the gating technique.

Figure 4 compares the performance of the TR-MUSIC pseudo-spectra using the non-gated MDM (conventional approach) and using the gated MDM (proposed approach). They were obtained by a single snapshot. In Fig. 4, the figures in the upper row represent the pseudo-spectra of TRMUSIC of the conventional approach, and the figures in the lower row represent the pseudo-spectra of TR-MUSIC of the proposed approach. The white squares represent the coordinates of the scatterers, that is, they show true positions. In addition, $D_{n}$ in the figure caption represents the number of dimensions of the noise subspace. The resulting imaging functions of the proposed approach yield more reliable images than the conventional approach for all simulation scenarios. Although the conventional approach cannot separate the two targets for 30 and $40 \mathrm{~dB}$, the proposed approach overcomes the problem by suppressing the noise component outside the gating region. This improves SNR, which produces better results of the TR-MUSIC algorithm. Similarly, Fig. 5 shows the images obtained using 10 snapshots. We can see that the images of the TR-MUSIC pseudo-spectra become stable as the number of snapshots increases. Like the preceding single snapshot case, the proposed approach offers more accurately estimated coordinates of the scatterers and more stable images than the conventional approach. It should be noted that the proposed approach can distinguish the two targets independently of these noise conditions (see the figures in the lower row in Fig. 5). From these results, it can be said that the proposed approach for TRMUSIC imaging reduces the noise component and provides accurate images. 


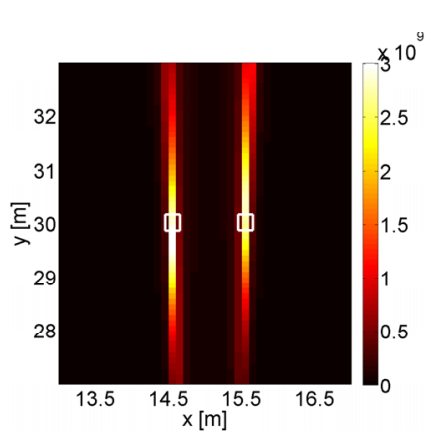

(a) $\mathrm{SNR}=60 \mathrm{~dB}$

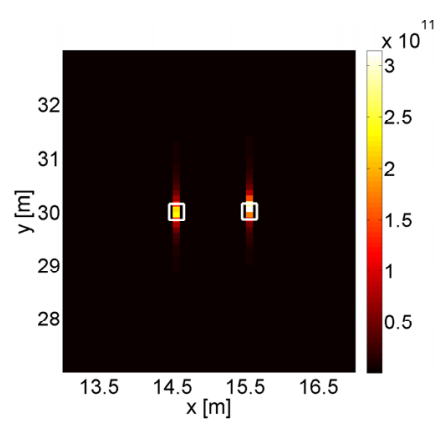

(e) $\mathrm{SNR}=60 \mathrm{~dB}$

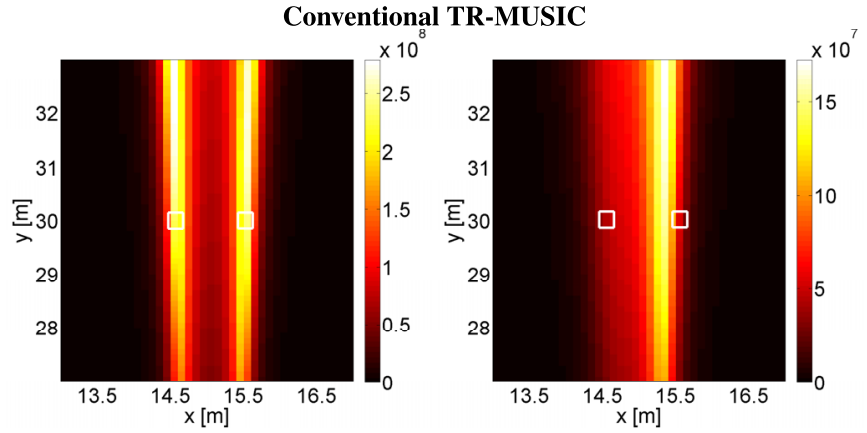

(b) $\mathrm{SNR}=50 \mathrm{~dB}$

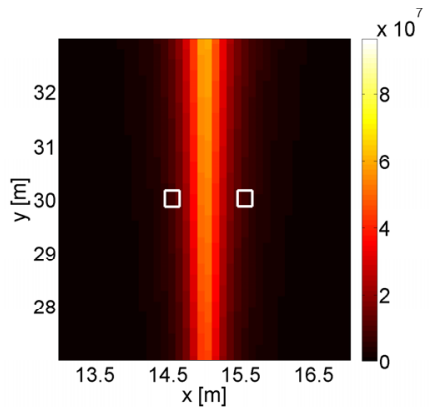

(d) $\mathrm{SNR}=30 \mathrm{~dB}$

\section{Proposed TR-MUSIC}

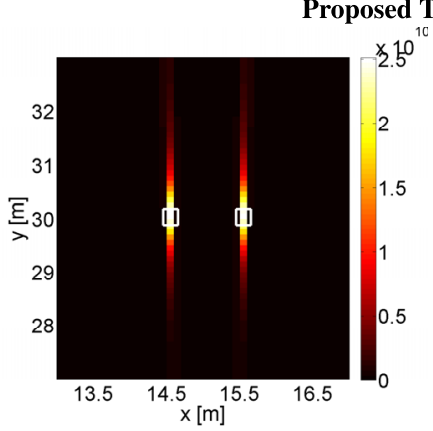

(f) $\mathrm{SNR}=50 \mathrm{~dB}$

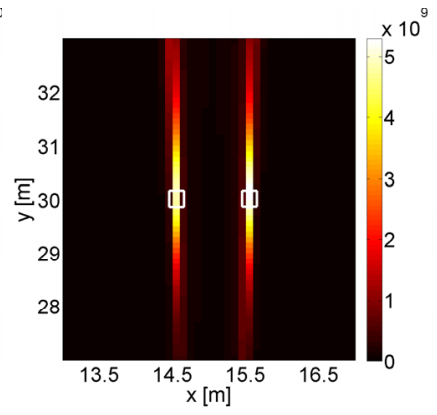

(g) $\mathrm{SNR}=40 \mathrm{~dB}$

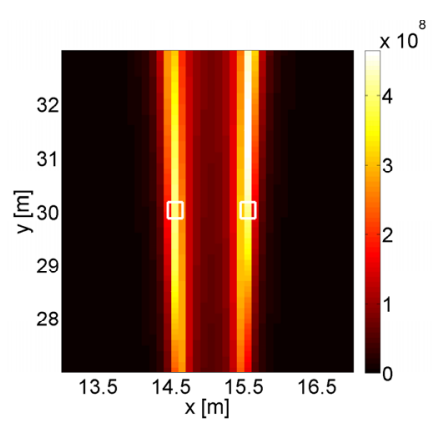

(h) $\mathrm{SNR}=30 \mathrm{~dB}$

Fig. 4 Images of the TR-MUSIC pseudo-spectra (1) (the number of snapshot $=1, D_{n}=9$ ). (a)-(d) conventional TR-MUSIC (e)-(h) proposed TR-MUSIC with gating.

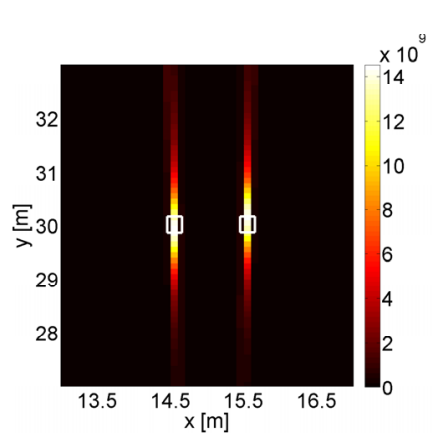

(a) $\mathrm{SNR}=60 \mathrm{~dB}$

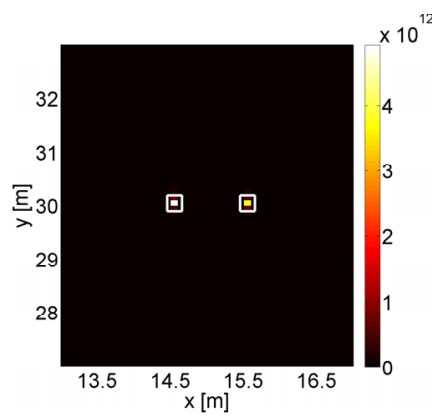

(e) $\mathrm{SNR}=60 \mathrm{~dB}$

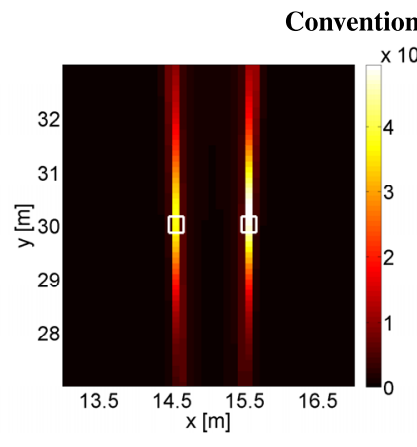

(b) $\mathrm{SNR}=50 \mathrm{~dB}$

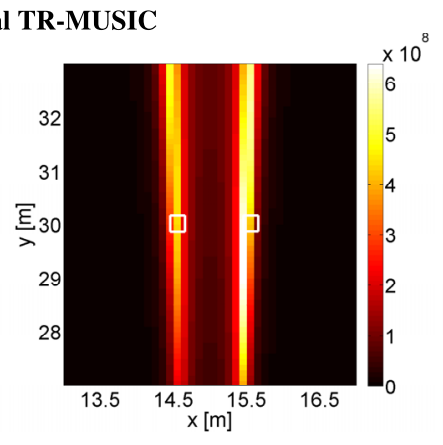

(c) $\mathrm{SNR}=40 \mathrm{~dB}$

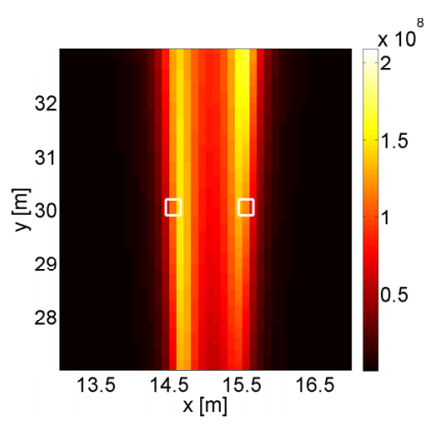

(d) $\mathrm{SNR}=30 \mathrm{~dB}$

Proposed TR-MUSIC

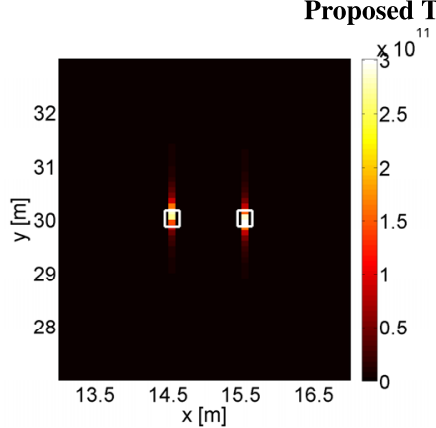

(f) $\mathrm{SNR}=50 \mathrm{~dB}$

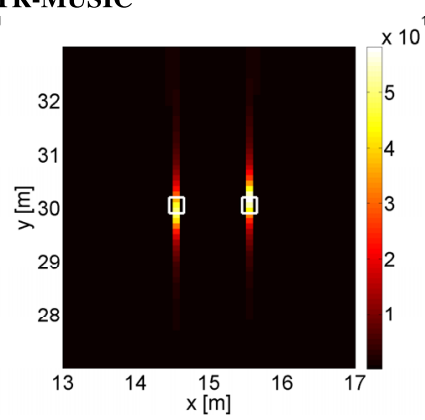

(g) $\mathrm{SNR}=40 \mathrm{~dB}$

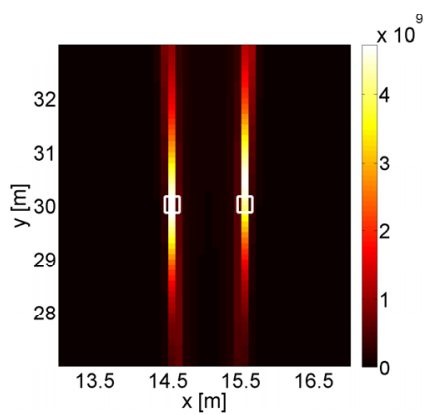

(h) $\mathrm{SNR}=30 \mathrm{~dB}$

Fig. 5 Images of the TR-MUSIC pseudo-spectra (2) (the number of snapshots $=10, D_{n}=9$ ). (a)-(d) conventional TR-MUSIC (e)-(h) proposed TR-MUSIC with gating. 


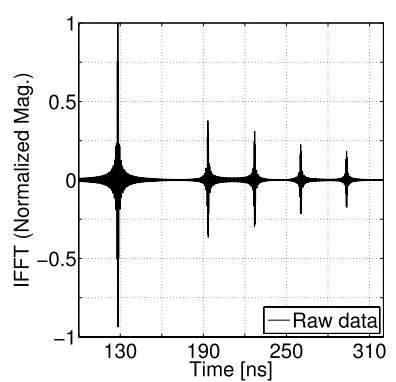

(a) Raw data

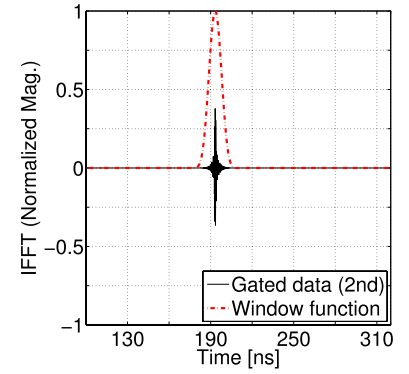

(b) Gated data (2nd target)

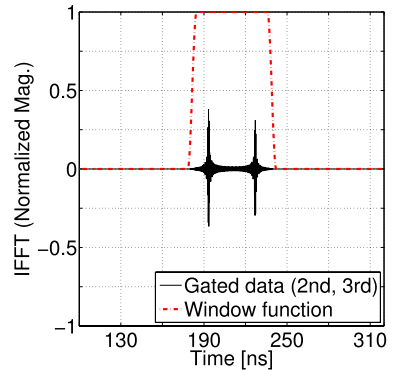

(c) Gated data (2nd and 3rd target)

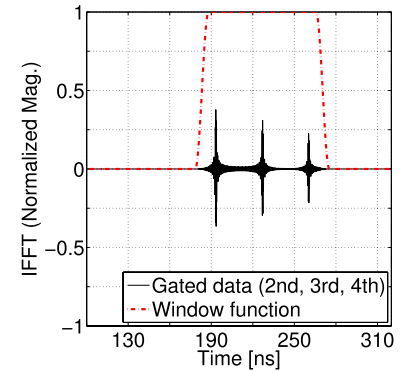

(d) Gated data (2nd, 3rd and 4th target)

Fig. 6 Extraction of targets in the time domain.

\subsection{Environment with More Targets than Antennas}

Next, we investigated the proposed approach in an environment where there are a greater number of scatterers than antenna elements $(N<M)$. To evaluate the algorithm's effectiveness, we used the following simulation scenario. The array had four antenna elements $(N=4)$ with $\lambda_{c} / 2$ spacing and it was placed parallel to the $\mathrm{x}$-axis. The first antenna was located at the coordinate $(15 \mathrm{~m}, 1 \mathrm{~m})$. Five scatterers $(M=5)$ located at $(18 \mathrm{~m}, 20 \mathrm{~m}),(15 \mathrm{~m}, 30 \mathrm{~m}),(17 \mathrm{~m}, 35 \mathrm{~m}),(13 \mathrm{~m}$, $40 \mathrm{~m})$, and $(16 \mathrm{~m}, 45 \mathrm{~m})$ had the same scattering coefficients $\rho_{m}=1(m=1,2,3,4$ and 5). Also, the imaging function for TR-MUSIC was obtained for the center frequency $f_{c}$. The other parameters, such as the frequency and noise variables, were the same as those in the previous subsection. We also considered an ideal noiseless scenario. The conventional TR-MUSIC imaging approach works only for $N>M$. For comparing performance, we considered the reference case when using the conventional TR-MUSIC approach in which the number of targets in the entire area is the same as that in the gated region, e.g., if we have two targets in the gated region, there are only the two targets in the entire region.

Figure 6 shows the time-domain response of $k_{1,1}$, gated responses, and window functions in an ideal noiseless environment. We can see the five peaks corresponding to their scatterers in Fig. 6(a) since the scatterers were located far apart. In addition, we observed the response attenuation due to the propagation loss obtained from the Green's function. In this simulation scenario, the gating process including four or more targets could not be used because we had only four antenna elements. For this reason, we carried out simulations for extracting a single target at $(15 \mathrm{~m}, 30 \mathrm{~m})$, two targets at $(15 \mathrm{~m}, 30 \mathrm{~m})$ and $(17 \mathrm{~m}, 35 \mathrm{~m})$ and three targets at $(15 \mathrm{~m}, 30 \mathrm{~m}),(17 \mathrm{~m}, 35 \mathrm{~m})$, and $(13 \mathrm{~m}, 40 \mathrm{~m})$. The extracted signals correspond to the second, third, and fourth responses in the time domain shown in Fig. 6(a). The gate parameters were determined according to the signal circumstances, as shown in Figs. 6(b), (c), and (d). In the single gated target, we had the gate center $T_{0}=194 \mathrm{~ns}$, the gate span $T_{g}=30 \mathrm{~ns}$, and the Raised Cosine window function with roll-off $=1$. Similarly, the gate parameters for extracting the two and three targets are as follows; (1) two targets: gate center $T_{0}=206 \mathrm{~ns}$, gate $\operatorname{span} T_{g}=64 \mathrm{~ns}$, and Raised
Cosine window function with roll-off $=0.2$, (2) three targets: gate center $T_{0}=227 \mathrm{~ns}$, gate span $T_{g}=97 \mathrm{~ns}$, and Raised Cosine window function with roll-off $=0.2$. As far as we have tried, the Raised Cosine window function was the most practical option for extracting multiple targets located apart without damaging the mainlobes. That is the reason why we employed it. Furthermore, we used different roll-off factors for the single and multiple targets. It has been found that if we use the same window function for these cases, the gated responses become seriously damaged. That is, if we apply the Raised Cosine window with roll-off $=1$ to the two-target case, the mainlobes of the 2 nd and 3 rd responses become seriously attenuated. Similarly, the mainlobes of the 2 nd and 4th responses become extremely reduced when we utilize the same window function and roll-off factor. In order to avoid this problem, we utilized the different roll-off factor $=$ 0.2 .

Figure 7 shows the TR-MUSIC pseudo-spectra of the targets. As can be seen from the discussions in Sect. 2, the noise subspace is the orthogonal complement of the signal subspace. Even in an ideal noiseless environment, we have the orthogonal complement of the signal subspace, and we consider it as the noise subspace. In this case, the eigenvectors in the noise subspace have the eigenvalue of 0 , and can be easily obtained. Unlike in the case in the preceding subsection, we added the 3-D imaging function of the TR-MUSIC pseudo-spectra. This is because 2-D images are very difficult to see in an ideal noiseless environment such as Figs. 7(c) and (f). In 2-D images, the white letters represent the position errors, that is, they show the error distances between the true target positions and the estimated coordinates having the peak value of the TR-MUSIC pseudo-spectra. In addition, the arrows in 3-D images show the true target positions, and the coordinates of them are shown numerically in the figures. As shown in Figs. 7(a)-(c) and (g)-(i), the reference case provides accurately estimated coordinates of the scatterers. Similarly, the proposed approach also reveals similar images in the single and two gated targets, as shown in Figs. 7(d), (e), (j), and (k). As for the two gated targets, one of the peaks had a lower value. On the other hand, the imaging function obtained from the three targets in the gated region degraded, as shown in Figs. 7(f) and (1). A time-domain signal response is in general comprised of one mainlobe, which contains the maximum power, and many 


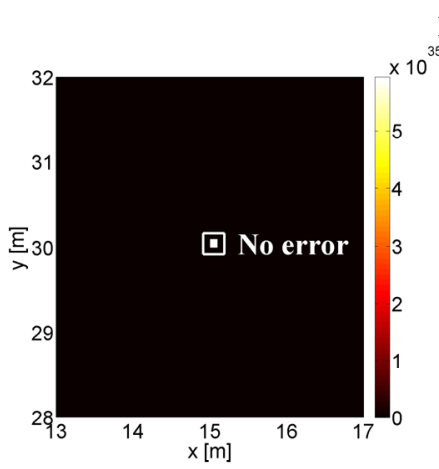

(a) Single target case $\left(D_{n}=3\right)$

Reference case (two dimensional image)

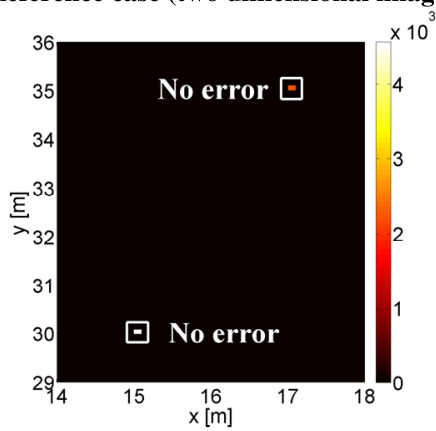

(b) Two target case $\left(D_{n}=2\right)$

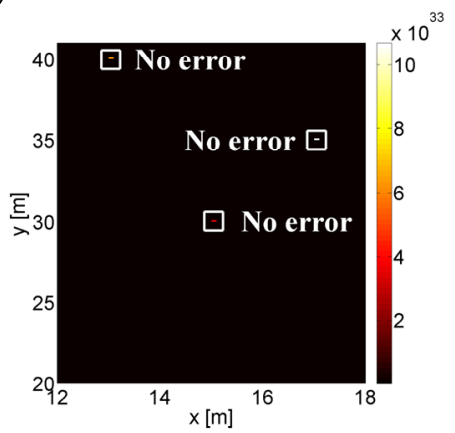

(c) Three target case $\left(D_{n}=1\right)$

Proposed scheme (two dimensional image)

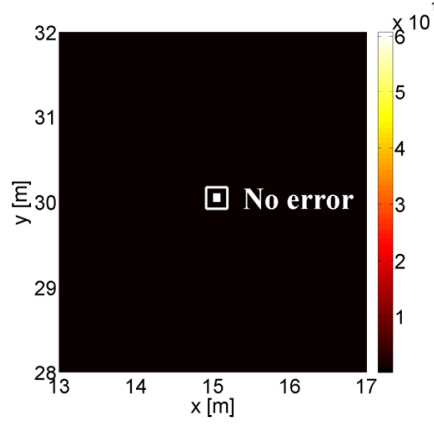

(d) Single target case $\left(D_{n}=3\right)$

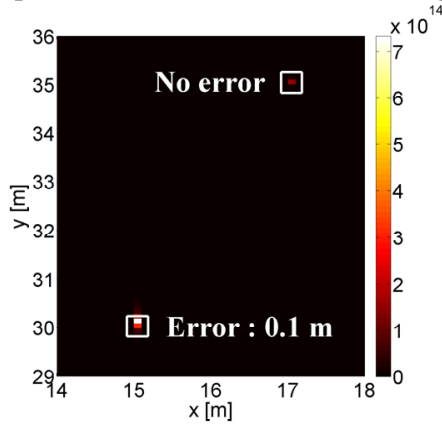

(e) Two target case $\left(D_{n}=2\right)$

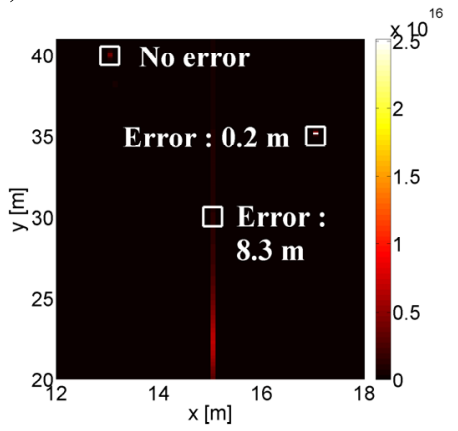

(f) Three target case $\left(D_{n}=1\right)$

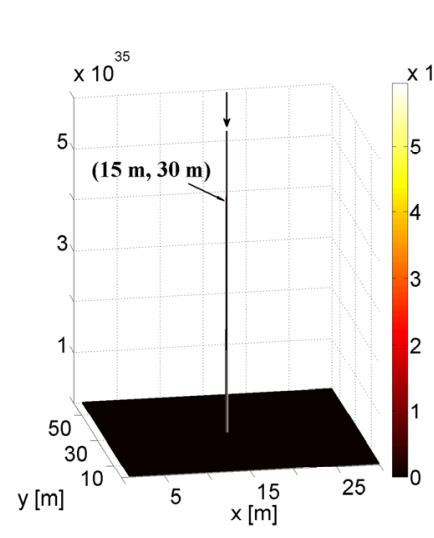

(g) Single target case $\left(D_{n}=3\right)$

Reference case (three dimensional image)

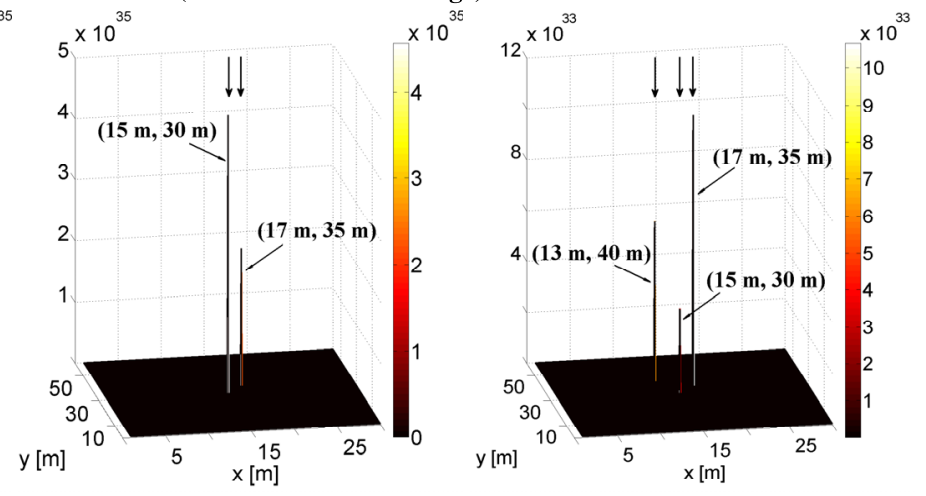

(h) Two target case $\left(D_{n}=2\right)$

(i) Three target case $\left(D_{n}=1\right)$

Proposed scheme (three dimensional image)

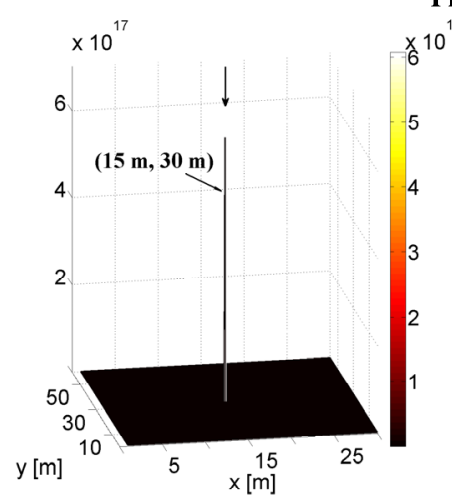

(j) Single target case $\left(D_{n}=3\right)$

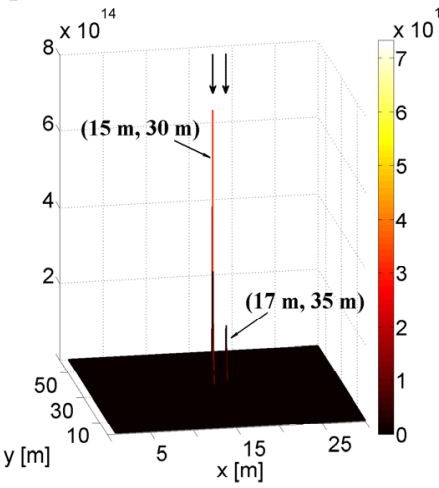

(k) Two target case $\left(D_{n}=2\right)$

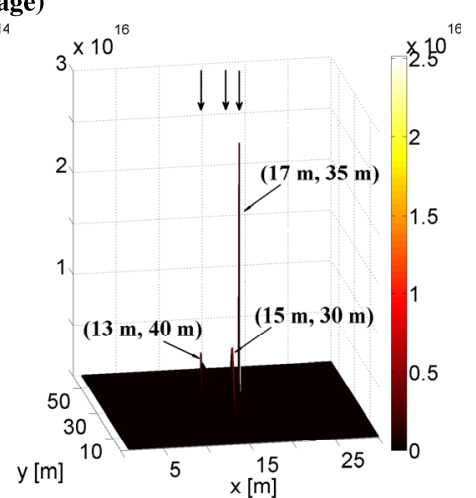

(l) Three target case $\left(D_{n}=1\right)$

Fig. 7 TR-MUSIC pseudo-spectra of targets. (a)-(c) TR-MUSIC with reference case (two dimensional image) (d)-(f) TR-MUSIC with proposed scheme (two dimensional image) (g)-(i) TR-MUSIC with reference case (three dimensional image) (j)-(1) TR-MUSIC with proposed scheme (three dimensional image). 


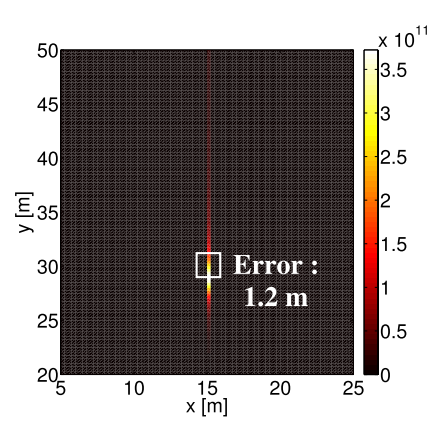

(a) $\mathrm{SNR}=60 \mathrm{~dB}$

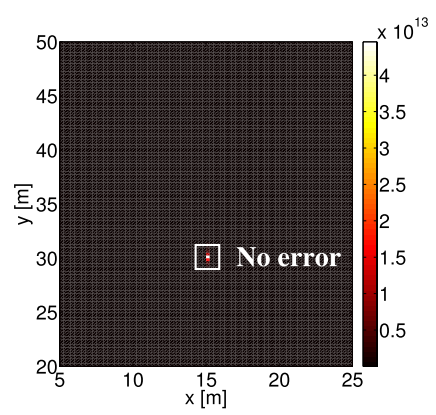

(e) $\mathrm{SNR}=60 \mathrm{~dB}$

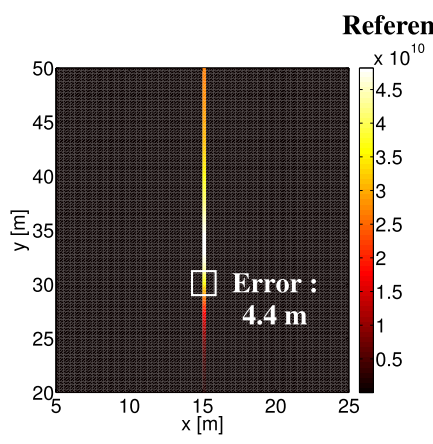

(b) $\mathrm{SNR}=50 \mathrm{~dB}$

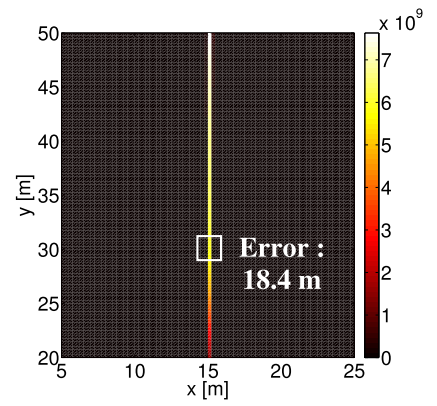

(c) $\mathrm{SNR}=40 \mathrm{~dB}$

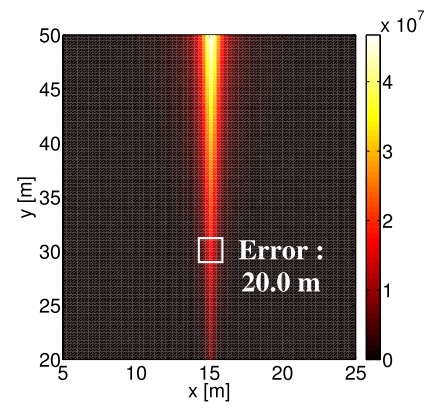

(d) $\mathrm{SNR}=30 \mathrm{~dB}$

Proposed scheme

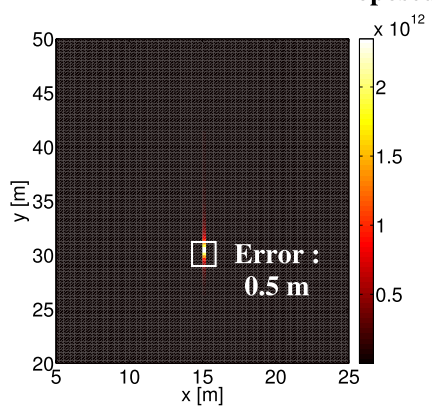

(f) $\mathrm{SNR}=50 \mathrm{~dB}$

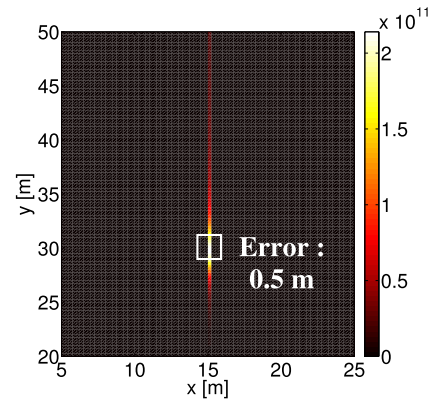

(g) $\mathrm{SNR}=40 \mathrm{~dB}$

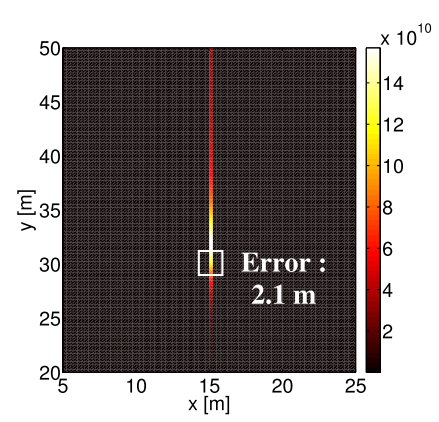

(h) $\mathrm{SNR}=30 \mathrm{~dB}$

Fig. 8 Images of the TR-MUSIC pseudo-spectra of the 2nd target (the number of snapshots $=10$, $D_{n}=3$ ). (a)-(d) TR-MUSIC with reference case (e)-(h) TR-MUSIC with proposed scheme.

sidelobes. Compared to the mainlobe, sidelobes contain relatively low power and they spread out over the entire region. The concept is similar to an antenna pattern. Although the time-domain gating technique can extract the desired responses without damaging the mainlobe of the signals [14][16], it may distort them slightly by removing the sidelobes. It is seen from Figs. 7(d), (e), and (f) (or (j), (k), and (l)) that the peak values of the TR-MUSIC pseudo-spectra for the proposed scheme are less than $10^{18}$. These values are much smaller than those for the reference case in which the TR-MUSIC pseudo-spectra have the peak values larger than $10^{32}$. It is conjectured that the reason why the peak values for the proposed scheme have smaller values is the effect of sidelobe suppression by the time-domain gating technique. However, what is important for the TR-MUSIC imaging is not peak values, but peak positions. Also, we believe that if there are sufficient noise subspace vectors, the minor loss due to the gating technique affects TR-MUSIC imaging less. As shown in Fig. 7, one- or two-dimensional noise subspace is not sufficient for TR-MUSIC imaging in this simulation scenario. On the other hand, we can clearly see that TRMUSIC reveals excellent imaging performance for the single gated target response. Thus, we discuss TR-MUSIC imaging for the single gated target in the following paragraph.

Figure 8 shows the performance comparison of the TRMUSIC pseudo-spectra in the reference case with those of the proposed approach in various noise environments for the second target at $(15 \mathrm{~m}, 30 \mathrm{~m})$. It should be noted that SNR is defined as the power ratio of the reflection from the second target to the noise. The proposed approach provides a more precisely estimated location of the scatterer and a clearer image than the reference case, as discussed in the preceding subsection. In particular, the reference images for SNRs of 40 and $30 \mathrm{~dB}$ (Figs. 8(c) and (d)) show deformed image functions with an erroneous coordinate, while the proposed approach demonstrates a stable image in the same SNR environments (Figs. 8(g) and (h)).

From these results, we can say that the proposed approach with the gating technique not only provides excellent performance but is also powerful in spite of a heavy noise environment with only a few snapshots.

\section{Conclusions}

We have proposed a time-reversal super-resolution imaging technique with the gating technique. Although the TRMUSIC algorithm was developed for the condition in which the number of transceivers is larger than that of targets, the proposed approach enables the algorithm to work even when this condition is violated. In addition, the proposed approach suppresses the noise components outside the gate (window) in the time domain. This leads to an increase in SNR and improves the imaging performance of TR-MUSIC.

With wider bandwidth data, we can further reduce the noise component due to a narrower gate span. However, a too narrow gate span destroys the signal component; therefore, the TR-MUSIC algorithm will not work properly. Optimum gating parameters should be studied in the future. 


\section{References}

[1] C. Prada, S. Manneville, D. Spoliansky, and M. Fink, "Decomposition of the time reversal operator: Detection and selective focusing on two scatterers," J. Acoust. Soc. Amer., vol.99, no.4, pp.20672076, April 1996

[2] M. Fink, D. Cassereau, A. Derode, C. Prada, P. Roux, M. Tanter, J. Thomas, and F. Wu, "Time-reversed acoustics," Rep. Prog. Phys., vol.63, no.12, pp.1933-1995, 2000.

[3] S.K. Lehman and A.J. Devaney, "Transmission mode time-reversal super-resolution imaging," J. Acoust. Soc. Am., vol.113, no.5, pp.2742-2753, May 2003.

[4] F.K. Gruber, E.A. Marengo, and A.J. Devaney, "Time-reversal imaging with multiple signal classification considering multiple scattering between the targets," J. Acoust. Soc. Am., vol.115, no.6, pp.3042-3047, June 2004.

[5] A.J. Devaney, "Time reversal imaging of obscured targets from multistatic data," IEEE Trans. Antennas Propag., vol.53, no.5, pp.16001610, May 2005

[6] D.H. Liu, G. Kang, L. Li, Y. Chen, S. Vasudevan, W. Joines, Q.H. Liu, J. Krolik, and L. Carin, "Electromagnetic time-reversal imaging of a target in a cluttered environment," IEEE Trans. Antennas Propag., vol.53, no.9, pp.3058-3066, Sept. 2005.

[7] A.J. Devaney, E.A. Marengo, and F.K. Gruber, "Time-reversal-based imaging and inverse scattering of multiply scattering point targets," J. Acoust. Soc. Am., vol.118, no.5, pp.3129-3138, Nov. 2005.

[8] M.E. Yavuz and F.L. Teixeira, "Full time-domain DORT for ultrawideband electromagnetic fields in dispersive, random inhomogeneous media," IEEE Trans. Antennas Propag., vol.54, no.8, pp.2305-2315, Aug. 2006.

[9] E.A. Marengo, F.K. Gruber, and F. Simonetti, "Time-reversal MUSIC imaging of extended targets," IEEE Trans. Image Process. vol.16, no.8, pp.1967-1984, Aug. 2007.

[10] M.E. Yavuz and F.L. Teixeira, "On the sensitivity of time-reversal imaging techniques to model perturbations," IEEE Trans. Antennas Propag., vol.56, no.3, pp.834-843, March 2008.

[11] M.E. Yavuz and F.L. Teixeira, "Space-frequency ultrawideband time-reversal imaging," IEEE Trans. Geosci. Remote Sens., vol.46, no.4, pp.1115-1124, April 2008.

[12] T. Sakamoto and T. Sato, "Time-reversal UWB imaging with a single antenna in multi-path environments,' EuCAP 2009, pp.21772181, Berlin, Germany, March 2009

[13] M.E. Yavuz and F.L. Teixeira, "Ultrawideband microwave sensing and imaging using time-reversal techniques: A review," Remote Sens., vol.1, pp.466-495, Aug. 2009.

[14] Y. Ogawa, T. Nakajima, H. Yamada, and K. Itho, "A superesolution technique for antenna pattern measurements," IEICE Trans. Commun., vol.E76-B, no.12, pp.1532-1537, Dec. 1993.

[15] H. Choi, Y. Ogawa, T. Nishimura, and T. Ohgane, "Noise reduction method based on the gating technique for time-reversal MUSIC imaging," IEEE iWEM 2011, pp.140-144, Taipei, Taiwan, Aug. 2011.

[16] H. Choi, Y. Ogawa, T. Nishimura, and T. Ohgane, "Challenge of time-reversal MUSIC imaging with a limited array," ISAP 2011, ThE1-2, Jeju, South Korea, Oct. 2011.

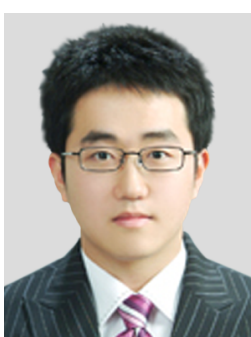

Heedong Choi received the B.S. degree in electronic engineering from Daegu University, Gyungsan, South Korea, in 2006, and the M.S degree in information and communication engineering from Yeungnam University, Gyungsan, South Korea, in 2008. Currently, he is a Ph.D. student in the Graduate School of Information Science and Technology in Hokkaido University, Sapporo, Japan. His research interests are in the area of signal processing for remote sensing and super-resolution technologies.

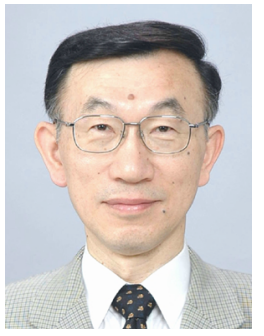

Yasutaka Ogawa received the B.E., M.E. and Ph.D. degrees from Hokkaido University, Sapporo, Japan, in 1973, 1975, and 1978, respectively. Since 1979, he has been with Hokkaido University, where he is currently a Professor of the Graduate School of Information Science and Technology. During 1992-1993, he was with ElectroScience Laboratory, the Ohio State University, U.S.A., as a Visiting Scholar, on leave from Hokkaido University. His interests are in adaptive antennas, mobile communications, super-resolution techniques, and MIMO systems. Dr. Ogawa received the Yasujiro Niwa outstanding paper award in 1978, the Young Researchers' Award of IEICE Japan in 1982, the Best Paper Award from IEICE Japan in 2007, and the Best Magazine Paper Award in 2011 from IEICE Communications Society. Dr. Ogawa is a Fellow of the IEEE.

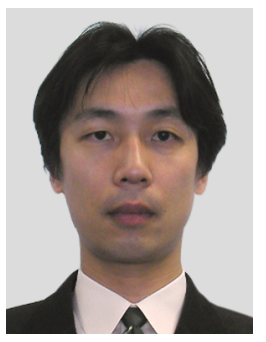

Toshihiko Nishimura received the B.S and M.S. degrees in physics and Ph.D. degree in electronics engineering from Hokkaido University, Sapporo, Japan, in 1992, 1994, and 1997 respectively. In 1998, he joined the Graduate School of Engineering (reorganized to Graduate School of Information Science and Technology at present) at Hokkaido University, where he is currently an Assistant Professor of the Graduate School of Information Science and Technology. His current research interests are in MIMO systems using smart antenna techniques. Dr. Nishimura received the Young Researchers' Award of IEICE Japan in 2000, the Best Paper Award from IEICE Japan in 2007, and the Best Magazine Paper Award in 2011 from IEICE Communications Society. Dr. Nishimura is a member of the IEEE.

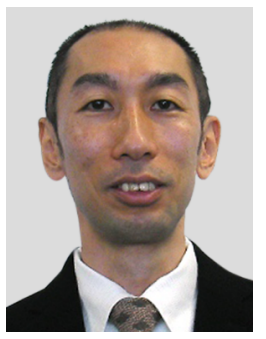

Takeo Ohgane received the B.E., M.E., and Ph.D. degrees in electronics engineering from Hokkaido University, Sapporo, Japan, in 1984, 1986, and 1994, respectively. From 1986 to 1992, he was with Communications Research Laboratory, Ministry of Posts and Telecommunications. From 1992 to 1995, he was on assignment at ATR Optical and Radio Communications Research Laboratory. Since 1995, he has been with Hokkaido University, where he is an Associate Professor. During 2005-2006, he was at Centre for Communications Research, University of Bristol, U.K., as a Visiting Fellow. His interests are in MIMO signal processing for wireless communications. Dr. Ohgane received the IEEE AP-S Tokyo Chapter Young Engineer Award in 1993, the Young Researchers' Award of IEICE Japan in 1990, the Best Paper Award from IEICE Japan in 2007, and the Best Magazine Paper Award in 2011 from IEICE Communications Society. Dr. Ohgane is a member of the IEEE. 